\title{
Coronavirus disease 2019 presenting with conjunctivitis as the first symptom
}

\author{
Giuseppe Casalino $\mathbb{D}^{1} \cdot$ Gaspare Monaco $^{1} \cdot$ Paolo Plinio Di Sarro ${ }^{1} \cdot$ Alessandro David $^{1} \cdot$ Antonio Scialdone $^{1}$
}

Received: 31 March 2020 / Revised: 15 April 2020 / Accepted: 16 April 2020 / Published online: 28 April 2020

(C) The Royal College of Ophthalmologists 2020

\section{To the Editor:}

Coronavirus disease 2019 (COVID-19) is a public health emergency that has reached the proportions of a pandemic [1]. At present, Italy has been one of the most affected Countries with the majority of cases documented in Northern Italy, especially in the Lombardia Region [2]. We herein report a case of confirmed COVID-19 presenting with unilateral conjunctivitis.

A 48-year-old man attended our Emergency Eye Department complaining of a 5-day history of redness and watery discharge in the right eye (RE) associated with foreign body sensation. Medical history was unremarkable with no history of cigarette smoking. On questioning, patient reported history of intermittent dry cough and mild fever over the last 3 days. Blood pressure was 135/90 mmHg. Temperature was $38^{\circ} \mathrm{C}$ and the oxygen saturation level was $97 \%\left(\mathrm{SpO}_{2}\right)$ while the patient was breathing ambient air. Clinical history and slit lamp examination were consistent with a viral conjunctivitis in the RE. Because of the respiratory symptoms and the ongoing COVID-19 outbreak in our Region, a chest X-ray scan was ordered and showed increased bronchovascular marking with no definite signs suggestive of pneumonia (Supplemental Figure). Nasal and pharyngeal swab specimens for real-time reverse transcriptase-polymerase chain reaction assay were taken and turned out to be positive for COVID-19. Patient was admitted for observation and

These authors contributed equally: Giuseppe Casalino, Gaspare Monaco

Supplementary information The online version of this article (https:// doi.org/10.1038/s41433-020-0909-x) contains supplementary material, which is available to authorized users.

Gaspare Monaco

gaspare.monaco@asst-fbf-sacco.it

1 Oftalmico Hospital, ASST Fatebenefratelli Sacco, Milan, Italy received supportive therapy consisting of oral paracetamol $1 \mathrm{~g}$ three times/day and a tapering course of topical tobramycin-dexamethasone in the RE. Given normalization of temperature and improvement of both the respiratory and the ocular symptoms, patient was discharged after 3 days of observation and home isolation for 14 days was advised.

Conjunctivitis seems to be a rare clinical finding among patients with confirmed diagnosis of COVID-19 since it has been reported in $<1 \%$ of the overall cases in China [3]. However, the presence of a mild conjunctivitis may have been underreported in those patients presenting with a complex and life-threatening clinical scenario.

While we cannot be entirely sure that the conjunctivitis in our case was a direct manifestation of COVID-19, the concomitance of the ocular and systemic flu-like symptoms makes the causal relationship likely. A recent study showed that COVID-19 nucleic acid may be detected in the tears and conjunctival secretions of patients with COVID-19-related pneumonia and conjunctivitis [4]. However, the accuracy of the conjunctival swab test has been questioned by the observation that patients with CoV-2-related conjunctivitis may have negative test results which, by contrast, may be positive in patients without conjunctivitis [5].

The importance of recognizing possible early ocular manifestation of COVID-19, as well as using protective eyewear among healthcare professionals, has already been outlined in recent editorials [5-7]. Since it has been speculated that the virus may enters the tears through droplets, which may pass through the nasolacrimal ducts, and then into the respiratory tract [4], conjunctivitis may represent one of the first symptoms of COVID-19. To the best of our knowledge, we report the first European confirmed COVID-19 case presenting with conjunctivitis as the first symptom. The present case outlines the importance of questioning patients presenting with conjunctivitis about respiratory and other systemic symptoms 
which should prompt clinicians to rule out COVID-19 infection.

Acknowledgements We thank all the colleagues and allied health professionals supporting our Eye Emergency Department and the other wards of the hospital during this difficult time of pandemic.

\section{Compliance with ethical standards}

Conflict of interest The authors declare that they have no conflict of interest.

Publisher's note Springer Nature remains neutral with regard to jurisdictional claims in published maps and institutional affiliations.

\section{References}

1. Callaway E, Cyranoski D, Mallapaty S, Stoye E, Tollefson J. The coronavirus pandemic in five powerful charts. Nature. 2020;579:482-3. https://doi.org/10.1038/d41586-020-00758-2.
2. Coronavirus COVID-19 Global Cases by the Center for Systems Science and Engineering at Johns Hopkins University. https://a rcgis.com/apps/opsdashboard/index.html\#/bda7594740fd40294923467b48e9ecf6. Accessed 30 Mar 2020.

3. Guan WJ, Ni ZY, Hu Y, Liang WH, Ou CQ, He JX, et al. China medical treatment expert group for Covid-19. Clinical characteristics of coronavirus disease 2019 in China. N Engl J Med. 2020. https://doi.org/10.1056/NEJMoa2002032.

4. Xia J, Tong J, Liu M, Shen Y, Guo D. Evaluation of coronavirus in tears and conjunctival secretions of patients with SARSCoV-2 infection. J Med Virol. 2020. https://doi.org/10.1002/jmv. 25725 .

5. Qing H, Li Z, Yang Z, Shi M, Huang Z, Song J, et al. The possibility of COVID-19 transmission from eye to nose. Acta Ophthalmol. 2020. https://doi.org/10.1111/aos.14412.

6. Li JO, Lam DSC, Chen Y, Ting DSW. Novel coronavirus disease 2019 (COVID-19): the importance of recognising possible early ocular manifestation and using protective eyewear. $\mathrm{Br} \mathrm{J}$ Ophthalmol. 2020;104:297-8.

7. Lu CW, Liu XF, Jia ZF. 2019-nCoV transmission through the ocular surface must not be ignored. Lancet. 2020;395:e39. https:// doi.org/10.1016/S0140-6736(20)30313-5. 